\title{
Successful Conservative Management of Neonatal MRSA Associated Tricuspid Valve Infective Endocarditis
}

\section{Sodhi MK ${ }^{1}$, Chhabra GS $^{2}$, Sarin SS $^{3}$}

${ }^{1}$ Dr. Manmeet Kaur Sodhi, MBBS, MD, Associate Professor, ${ }^{2}$ Dr. Gurpreet Singh Chhabra, MBBS, MD, Associate Professor, Paediatrics, ${ }^{3}$ Dr. Simarjot Singh Sarin, MBBS, MD, DM, Assistant Professor Consultant Cardiologist. All from Sri Guru Ram Das Institute of Medical Sciences and Research, Sri Amritsar, Punjab, India.

\section{Introduction}

W ith the advent of neonatal intensive care and use of long IV-lines, especially for parenteral nutrition, more and more cases of IE are diagnosed. Neonates with IE usually do not have underlying structural heart anomalies. All neonates with persistent or recurrent sepsis in an intensive care setting should be worked up for IE. A high index of suspicion, early diagnosis and treatment may affect the outcome favourably. This case is appropriate for reporting as this is a rare successful management of neonatal endocarditis on conservative therapy as the mortality in most cases is very high.

\section{The Case}

The patient was a full term baby boy born to a 26 year old G2P1 mother by normal vaginal delivery at Civil Hospital, Amritsar. There was history of delayed cry with APGAR score of 7 at birth. The baby was immediately admitted in NICU at Government Medical College, Amritsar and treated with antibiotics (cefotaxime and amikacin). No evidence of sepsis was found on peripheral blood film, complete blood count and CRP. Blood and urine culture was not sent and lumbar puncture was not performed. The baby was discharged on day 5 of life in a satisfactory condition. For the next five days; the baby's stay at home was uneventful. But then, the baby was re-admitted at the same NICU with probable sepsis. The baby was again given a 5-day course of antibiotics (cefotaxime and amikacin). The haemogram and Peripheral Blood Film (PBF) were normal. CSF, $\mathrm{X}$-ray chest and cultures from blood and urine were not

\begin{abstract}
Prognosis in neonatal endocarditis depends on early diagnosis and the status of the patient at the time of diagnosis, and effective treatment with appropriate antibiotics. The current case report describes a case of successful conservative treatment of endocarditis in a 26 day old male neonate, previously inadequately treated for septicaemia in another hospital.
\end{abstract}

Key words: Endocarditis, Neonatal

done for the second time. The baby was discharged on day 16 of life in a clinically stable condition.

The baby reported to our NICU at SGRD Hospital and Medical College, Amritsar on day 26 of life with seizures and fever. On examination at the time of admission, the neonate had heart rate of $142 / \mathrm{min}$, regular, respiratory rate of $58 / \mathrm{min}$ and $\mathrm{CFT}<3 \mathrm{sec}$. The neck was kept retracted, but the anterior fontanelle was at level. Neurological examination showed increased tone and normal reflexes. CVS examination revealed a systolic murmur over parasternal area. There were no congenital abnormalities and systemic examination including fundus examination was normal.

Investigations revealed $\mathrm{Hb}-4.5 \mathrm{gm} \%$, total leukocyte count-23000, DLC-64/33/2/1.The peripheral blood film showed toxic granules $3+$ with $15 \%$ band forms, suggesting septicaemia. Blood culture grew methicillin resistant staphylococcus aureus (MRSA) with sensitivity to gentamycin, amikacin, vancomycin. The CSF and urine examination was normal. Echocardiographic apical four chamber view showed one large $10 \mathrm{~mm} \times$ $10 \mathrm{~mm}$ vegetation attached to the atrial side of posterior leaflet of tricuspid valve. [Figure 1].

The baby was started on antibiotics (vancomycin and piperacillin) and intravenous fluids from day one. On the second day, the patient developed signs and symptoms of congestive cardiac failure in the form

\section{How to cite this article?}

Sodhi MK, Chhabra GS, Sarin SS. Successful Conservative Management of Neonatal MRSA Associated Tricuspid Valve Infective Endocarditis. J Nepal Paediatr Soc 2013;33(1):70-73. 
of tachypnoea, tachycardia, gallop rhythm, systolic murmur, increase in liver size and respiratory distress, and so he was ventilated for two days. Thereafter the baby improved and after one week of antibiotics, he was started on nasogastric feeding. He received intravenous antibiotics for a total of four weeks. Repeat blood culture taken after seven days of starting antibiotics was negative. One month later, (Figure 3), the apical four chamber view of echocardiography showed very small residual/healed vegetation attached over the posterior tricuspid leaflet. Colour flow echocardiography also showed severe tricuspid regurgitation with concentric jet flow towards interatrial septum on apical four chamber view. (Figure 4)

Three months after the successful conservative treatment, the baby on follow up was observed to have gained adequate weight, had normal CVS examination and age appropriate neurological development.

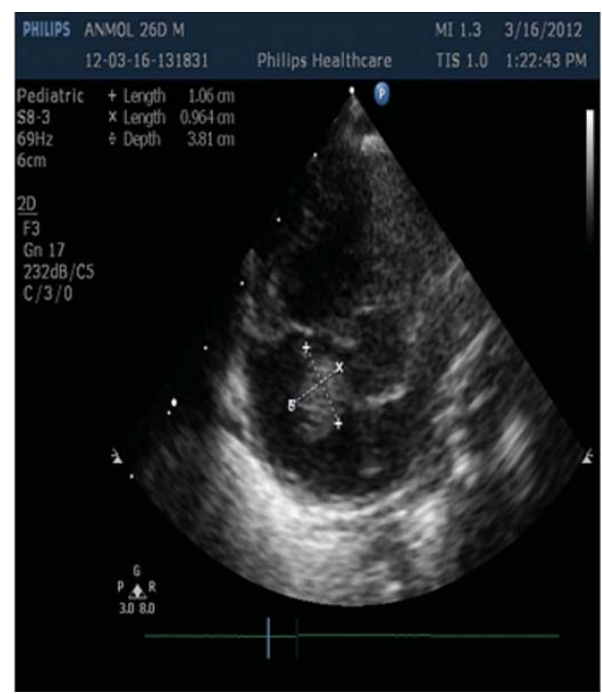

Fig 1: Echo showing $10 \times 10 \mathrm{~mm}$ vegetation on the posterior leaflet of tricuspid valve.

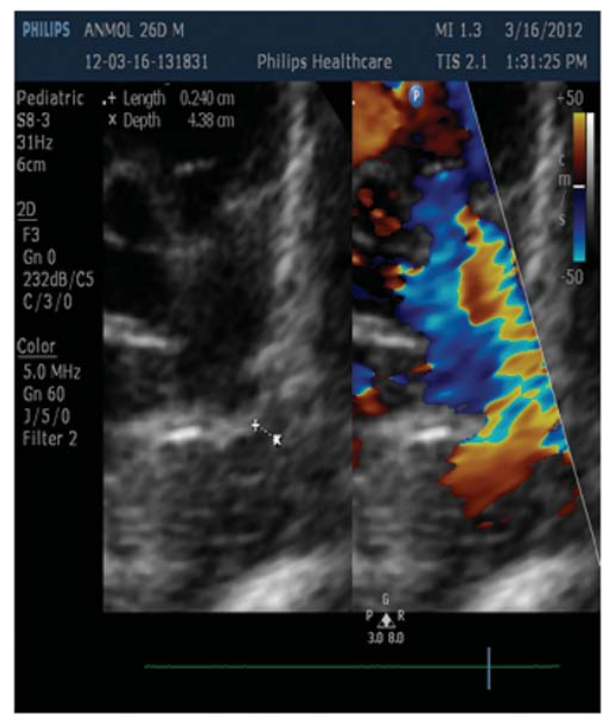

Fig 2: Parasternal view showing small PDA

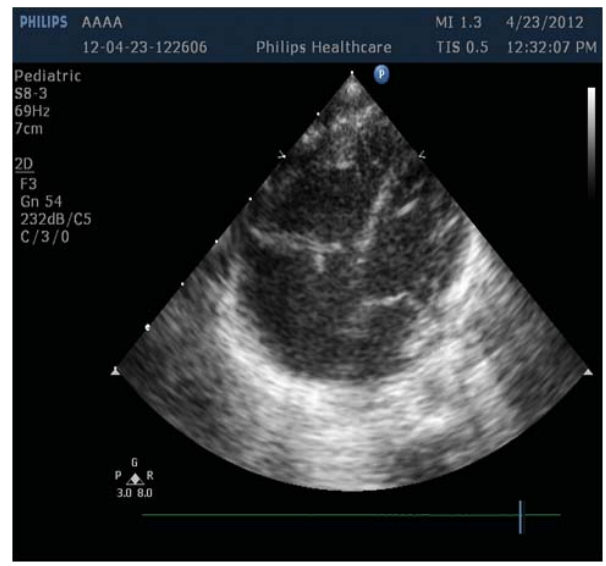

Fig 3: Echo showing small/residual healed vegetation.

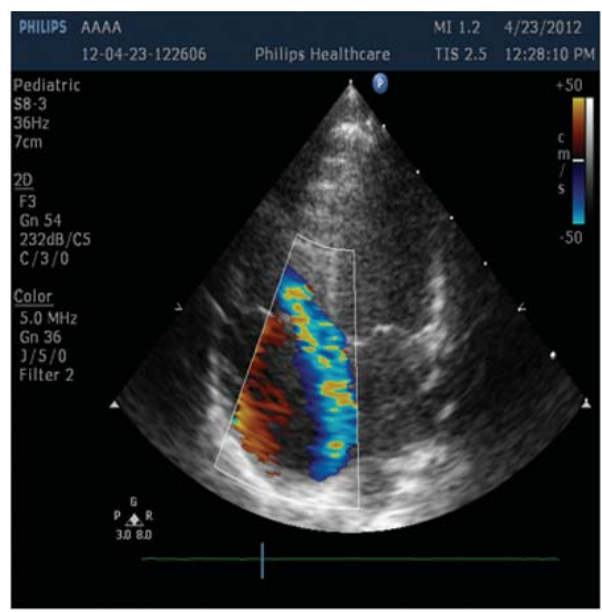

Fig 4: Echo showing severe tricuspid Regurgitation.

\section{Discussion}

Congenital heart disease is found in only $8 \%$ of neonates with infective endocarditis, compared to $80 \%$ in older children and adults ${ }^{1}$. This prevalence of infective endocarditis (IE) in infants with normal hearts is partly related to the increased frequency of sepsis in these infants. When IE develops in newborn infants, it is associated with a very high mortality rate; the diagnosis is often made at post-mortem examination ${ }^{2,3,4}$. However, with rapidly improving imaging technology and increasing clinical experience, the ante mortem diagnosis of neonatal IE is being made with much greater facility than in the past, and the incidence of neonatal endocarditis may be increasing ${ }^{2}$.

Neonatal endocarditis frequently occurs on the right side of the heart and is associated with disruption of endocardium or valvular endothelial tissue produced by catheter-induced trauma. Neonates often experience transient episodes of bacteraemia from trauma to the skin and mucous membranes, vigorous endotracheal suctioning, parenteral hyper alimentation, or placement of umbilical or peripheral venous catheters ${ }^{5}$. Considerable data support the notion that the interactions of Gram- 
positive cocci with platelets and the organism's capacity to resist the antimicrobial host defence properties of platelets are pivotal in the production and persistence of endocardial infections ${ }^{6}$. In our patient, there is history of previous hospitalization during which parental alimentation and peripheral cannulation did occur.

The clinical features of IE in a neonate are variable, nonspecific and indistinguishable from septicaemia, ${ }^{2,3,4}$ as was also observed in the present case report. Septic embolic phenomena are common, resulting in osteomyelitis, meningitis, or pneumonia. Neonates with IE often have feeding difficulties, respiratory distress, tachycardia, a new or changing heart murmur and hypotension. Many neonates with IE have neurological signs (seizures, hemiparesis, or apnoea). Arthritis, Osler nodes, Roth's spots, Janeway lesions, and splinter haemorrhages have not been described in neonates ${ }^{7}$.

Most organisms that cause IE in children are Gram positive cocci, streptococci, staphylococci, and enterococci. The rarity of IE caused by Gramnegative bacilli is due to their poor adhesion to cardiac valves. Paediatric patients who inject drugs intravenously are at risk for IE caused by $S$ aureus, as was seen in our patient. Fungal endocarditis usually is caused by Candida species, although Aspergillus has been reported too. With the use of central venous catheters and high glucose concentrations and hyper alimentation, Candida infections of the mural or valvular endocardium in infants have been widely recognized ${ }^{8}$. Fungal endocarditis also often is associated with very large friable vegetations; emboli from these vegetations frequently produce serious complications.

Two-dimensional echocardiography has become the main modality for detecting endocardial infection. In fact, certain echocardiographic findings are included as major criteria in the Duke criteria ${ }^{9}$. Typical echo-Doppler findings include vegetations, abscesses, new valvular insufficiency, and other acute changes in intracardiac flow patterns. The hallmark echocardiographic finding - the vegetation-may not always be visible with transthoracic echocardiography (TTE $)^{10}$. With a reported sensitivity of $81 \%,{ }^{11}$ TTE is more sensitive in the paediatric population than in the adult population for detection of vegetation. Certain echocardio-graphic features seem to be associated with complications which include large vegetation size $(>1 \mathrm{~cm})$, size that increases during therapy, and marked changes in Doppler evidence of worsening valvular or ventricular function ${ }^{12}$.

In infants, intravenous antibiotics are preferred over intramuscular agents because of the patients' small muscle mass. Outpatient treatment of endocarditis can be considered in selected patients who are hemodynamically stable and afebrile, have negative blood cultures, and are not at high risk for complications. Bacteraemia generally resolves within several days after appropriate therapy has begun; $S$ aureus bacteraemia may persist for 3 to 5 days with beta-lactam antistaphylococcal therapy and for 5 to 10 days with vancomycin therapy. Blood cultures should be repeated to assess the adequacy of treatment and to document the cessation of bacteremia. Additional blood cultures should be performed once or twice in the 8 weeks after completion of antibiotic treatment to ensure cure ${ }^{13}$. Antibiotics are needed in a higher concentration than with routine sepsis, because they have to penetrate the vegetation to reach the enmeshed bacteria. Still the mortality reported at present is $25 \%$ $60 \%$, but could possibly be decreased further if early diagnosis is made ${ }^{14}$.

Common indications for surgery include progressive cardiac failure, valvular obstruction, perivalvular extension of infection, fungal endocarditis, persistent bacteraemia despite appropriate antibiotic therapy and significant embolic events, especially when the aortic or mitral valve is involved ${ }^{15}$.

\section{References}

1. Callaghan $\mathrm{O}, \mathrm{McD}$ ouglie $\mathrm{P}$. Infective endocarditis in neonates. Arch Dis Child 1988;63:53-7.

2. Millard DD, Shulman ST. The changing spectrum of neonatal endocarditis. Clin Perinatol 1988;15:587608.

3. Oelberg DG, Fisher DJ, Gross DM, etal. Endocarditis in high-risk neonates. Pediatrics 1983;71:392-97.

4. Symchych PS, Krauss AN, Winchester P. Endocarditis following intracardiac placement of umbilical venous catheters in neonates. $J$ Pediatr 1977;90:287-89.

5. Picarelli D, Leone R, Duhagon P, Peluffo C, Zúñiga C, Gelós S,et al. Active infective endocarditis in infants and childhood: Ten year review of surgical therapy. J Card Surg 1997;12:406-11.

6. Rastogi A, Luken JA, Pildes RS, Chrystof D, LaBranche F. Endocarditis in Neonatal Intensive Care Unit. Pediatr Cardiol 1993;14:183-6.

7. Daher $\mathrm{AH}$. Infective endocarditis in neonates. Clin Pediatr 1995;4:198-206.

8. Fukushige J, Igarashi $\mathrm{H}$, Ueda K. Spectrum of infective endocarditis during infancy and childhood. Pediatric Cardiol 1994;15:127-31.

9. Durack DT, Lukes AS, Bright DK. New criteria for diagnosis of infective endocarditis: utilization of specific echocardiographic findings. Duke Endocarditis Service. Am J Med 1994;96:200-209. 
10. Bricker JT, Latson LA, Huhta JC, et al. Echocardiographic evaluation of endocarditis in children. Clin Pediatr (Phila). 1985;24:312-17.

11. Kavey RE, Frank DM, Byrum CJ, et al. Twodimensional echocardiographic assessment of infective endocarditis in children. Am J Dis Child 1983;137:851-56.

12. Vuille $C$, Nidorf $M$, Weyman AE, et al. Natural history of vegetations during successful medical treatment of endocarditis. Am Heart J 1994;128:1200-209.
13. Santoro J, Ingerman M. Response to therapy: relapses and reinfections. In: Kaye D, ed. Infective Endocarditis. 2nd ed. New York, NY: Raven Press; 1992:423-434.

14. Mecrow IK, Ladusans EJ. Infective endocarditis in newborn infants with structurally normal heart. Acta Paediatrica 1994;83:35-9.

15. Citak M, Rees A, Mavroudis C. Surgical management of infective endocarditis in children. Ann Thorac Surg 1992;54:755-60. 